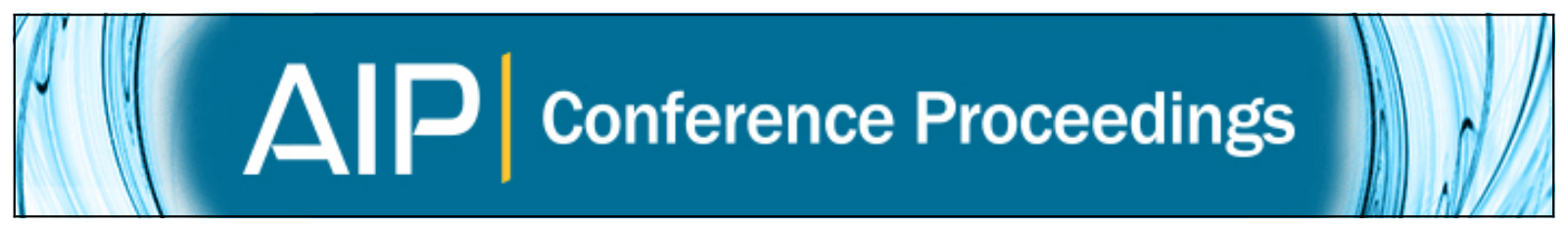

\title{
Physics based calibration of the Herschel/SPIRE bolometers
}

Adam L. Woodcraft, Hien Nguyen, James Bock, Matthew Griffin, Bernhard Schulz, Bruce Sibthorpe, and Bruce

Swinyard

Citation: AIP Conference Proceedings 1185, 95 (2009); doi: 10.1063/1.3292458

View online: http://dx.doi.org/10.1063/1.3292458

View Table of Contents: http://scitation.aip.org/content/aip/proceeding/aipcp/1185?ver=pdfcov

Published by the AIP Publishing

\section{Articles you may be interested in}

A distributed-absorber cold-electron bolometer single pixel at $95 \mathrm{GHz}$

Appl. Phys. Lett. 107, 092602 (2015); 10.1063/1.4929604

Observing Cold Dust with Herschel / SPIRE

AIP Conf. Proc. 1240, 89 (2010); 10.1063/1.3458571

Tuning of Kilopixel Transition Edge Sensor Bolometer Arrays with a Digital Frequency Multiplexed Readout System

AIP Conf. Proc. 1185, 253 (2009); 10.1063/1.3292326

Optical Properties of Argonne/KICP TES Bolometers for CMB Polarimetry

AIP Conf. Proc. 1185, 203 (2009); 10.1063/1.3292315

Development of high-sensitivity bolometers at CEA: preparing the PACS successors

AIP Conf. Proc. 1185, 119 (2009); 10.1063/1.3292297 


\title{
Physics based calibration of the Herschel/SPIRE bolometers
}

\author{
Adam L. Woodcraft ${ }^{*} \dagger$, Hien Nguyen ${ }^{* *}$, James Bock**, Matthew Griffin ${ }^{\ddagger}$, Bernhard \\ Schulz $^{\S}$, Bruce Sibthorpe ${ }^{\dagger}$ and Bruce Swinyard ${ }^{ף}$ \\ ${ }^{*}$ SUPA, Institute for Astronomy, University of Edinburgh, Blackford Hill, Edinburgh EH9 3HJ, UK \\ ${ }^{\dagger}$ UK Astronomy Technology Centre, Blackford Hill, Edinburgh EH9 3HJ, UK \\ ** Jet Propulsion Laboratory, 4800 Oak Grove Drive, Pasadena, CA 91109, USA \\ ${ }^{\ddagger}$ Cardiff School of Physics and Astronomy, Cardiff University, The Parade, Cardiff CF24 3AA, UK \\ $\S$ Infrared Processing and Analysis Center, California Institute of Technology, Pasadena, CA 91125, USA \\ "Rutherford Appleton Laboratory, STFC, Chilton, Didcot, Oxon, OX11 OQX, UK
}

\begin{abstract}
The bolometers (and readout circuitry) in the SPIRE instrument on the Herschel Space Observatory are among the best understood and well characterised of any sub-mm astronomy instrument to date. SPIRE contains five arrays of NTD germanium spiderweb bolometers with up to 139 pixels per array. Their behaviour has been shown to be extremely stable as seen by repeated measurements in the years between initial array level and final instrument level tests, and can be described extremely well by a simple physical model (the ideal bolometer model). Calibration of the bolometers must take into account the non-linear response when viewing bright sources, and the effect of fluctuations in the heat sink temperature. The simple and well-understood behaviour of the detectors, coupled with the stable conditions expected in flight, mean that in contrast to previous sub-mm instruments, physical models can be used to improve or possibly replace empirical calibration methods. We describe how this can be done, and use the large amount of data from ground measurements to show that we can use models to accurately calculate the absolute power detected by the bolometers.
\end{abstract}

Keywords: Sub-mm astronomy, bolometers, NTD germanium, calibration, Herschel, SPIRE PACS: $95.55 . \mathrm{Fw}, 95.55 . \mathrm{Rg}$

\section{INTRODUCTION}

SPIRE (the Spectral and Photometric Imaging Receiver) is one of three instruments on board the Herschel Space Observatory [1], an ESA mission with NASA participation, launched in May 2009. This paper describes measurements on the three arrays in the SPIRE photometer; these have bands centred at approximately 250,350 and $500 \mu \mathrm{m}$ (referred to in this paper as the PSW, PMW and PLW arrays respectively). A further two arrays make up the spectrometer. The arrays, designed and constructed at the Jet Propulsion Laboratory (JPL) in the US, consist of feedhorn-coupled NTD (neutron transmutation doped) germanium spider-web bolometers cooled to $300 \mathrm{mK}$ by a closed cycle ${ }^{3} \mathrm{He}$ refrigerator.

Bolometers are - in principle - extremely simple devices [2]. Figure 1 shows the operating principles of a

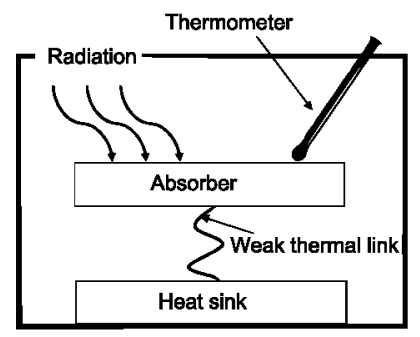

FIGURE 1. Schematic layout of a bolometer. bolometer. For a bolometer using a resistive thermometer, the static behaviour can be described fully by the relationship between thermistor resistance and temperature $(R(T))$, and the thermal conductance of the weak thermal link between the absorber and the heat sink $(G(T))$. For the SPIRE bolometers, we can take these as:

$$
R(T)=R^{*} \exp \sqrt{T_{g} / T}, \quad G(T)=G_{0} T^{\beta} .
$$

Together, these two equations are known as the ideal (bolometer) model, and in order to predict the behaviour of the bolometer, all we need to know is the four parameters $R^{*}, T_{g}, G_{0}$ and $\beta$ (in addition, we need the optical efficiency if we wish to work in terms of incident rather than absorbed power). The parameters $G_{0}$ and $\beta$ can be determined from measurements under optical load, but "blanked measurements", in which there is negligible optical load, are required to unambiguously determine $R^{*}$ and $T_{g}$.

We were able to carry out extensive ground tests on SPIRE, using the flight electronics, with the bolometers both viewing optical radiation (run "PFM4") and with a cover over the bolometers to give blanked measurements ("PFM5"). During the optical run, the bolometers were illuminated by a cryogenic black-body (CBB) which could be heated to temperatures up to $15 \mathrm{~K}$. We have shown previously [3] that these tests demonstrate that the SPIRE bolometers are described extremely well by 
the ideal model, and that the bolometers and read-outs are extremely stable. A consequence of this is that we could consider using the bolometer model to calibrate the bolometers.

Traditionally, calibration of sub-mm instruments is carried out empirically, by measuring the change in power corresponding to a given change in bolometer voltage. This is a linear relationship to a good approximation, but nonlinearity becomes significant for bright sources, and must be measured. There are many good reasons for using empirical calibrations. Until recently, bolometer instruments generally used DC readouts. This results in an unknown offset in voltage. While this does not matter when measuring voltage changes, the lack of knowledge of the absolute voltage prevents the model from being applied. Other obstacles to using the bolometer model for calibration are that it requires extremely good stability, precise characterisation, and - of course - bolometers which follow the ideal model (this is generally not the case for semiconductor bolometers designed for use at $100 \mathrm{mK}$ ).

Currently, SPIRE will also use an empirical calibration, since this is known to work. In principle, a model based calibration should also work. However, there are many reasons why in practice it may not. For example, if the readout electronics were to introduce a small but slowly varying offset in measured bolometer voltage, this would have no effect on a standard empirical calibration, but could cause large errors in a model based calibration. The success of the model therefore depends on either near perfect behaviour, or on methods being found to compensate for less than perfect behaviour. Here, we use ground-based measurements to examine whether a model based calibration might work.

\section{ANALYSIS}

The results shown here are all derived from "load curve" measurements; i.e. measurements of bolometer voltage as a function of bias current. We can derive the difference in absorbed power between two load curves without any knowledge of bolometer parameters, by comparing the electrical power at the same bolometer resistance for the two load curves. These points must be at the same temperature, and therefore must have the same total power flowing down the weak thermal link. We can calculate the electrical power dissipated in the absorber, and the remaining power must therefore be absorbed optical power. Such results are shown in Fig. 2. The derived power is constant with detector temperature to a good approximation. This should of course be the case, but such behaviour is not always seen. The dark pixels are bolometers identical to the other pixels, except that they are blocked from optical power. The power

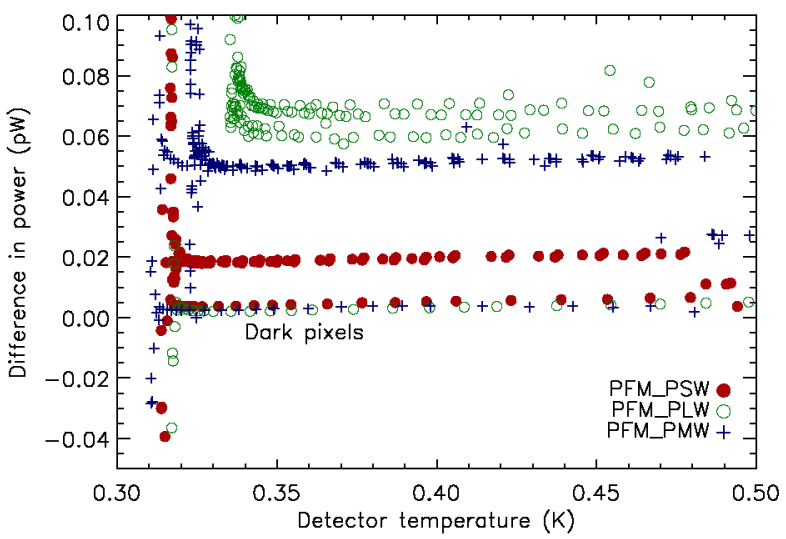

FIGURE 2. Difference in optical power for a few bolometers from each array, calculated from pairs of load curves under different optical loads, without using any model parameters.

difference for these pixels is slightly larger than zero, due to a small change in heat sink temperature between the measurements (such changes may also be present during flight).

This is a disadvantage of this type of calculation. However, the main disadvantage is that in normal operation we do not carry out load curves; we only measure the bolometer voltage at a particular value of bias current (the operating bias). Consequently, this method cannot be used in normal operation, though load curves on calibration sources may provide extremely useful information. Instead, we must use the ideal model to predict the power required to raise the absorber to the measured temperature. To do this we need to know the temperature of the bolometer heat sink. This can be determined from thermistors on the array, but we obtain better results from the dark bolometers, using the bolometer model to deduce the heat sink temperature from the absorber temperature.

Results from this process are shown in Fig. 3. Again, the optical power remains constant with temperature, showing the model is performing extremely well. As would be expected, the dark pixel (DK2) shows no change with optical power. In Fig. 4 we show the performance of all the bolometers. From here onwards, we simulate regular observations by only using the bolometer voltage at a fixed bias, rather than entire load curves; Fig. 3 shows that it does not matter what bias value we use (in a load curve, absorber temperature increases monotonically with bias, so if Fig. 3 were plotted with bias on the $x$-axis instead of temperature it would look similar). Each panel in Fig. 4 shows the agreement between change in power from the $\mathrm{CBB}$ at two $\mathrm{CBB}$ temperatures $\left(\triangle P_{C B B}\right)$, calculated from the $\mathrm{CBB}$ temperature, and the change in power determined from the bolometer model $\left(\Delta P_{\text {meas }}\right)$, in the form $\left(\Delta P_{\text {meas }} / \Delta P_{C B B}\right)$. The $\mathrm{CBB}$ is known to provide non-uniform illumination, and the results are therefore normalised by the ratio of the mea- 


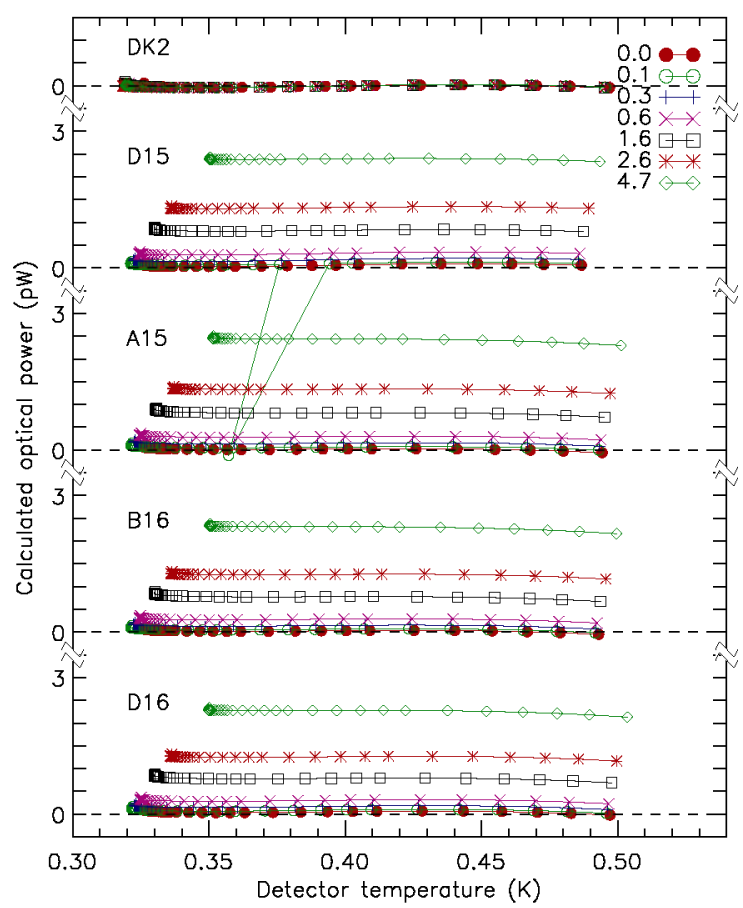

FIGURE 3. Optical power derived from the model parameters as a function of absorber temperature for a few PSW array pixels at different CBB powers (shown in the key, in $\mathrm{pW}$ ).

sured and calculated power with the blackbody at $15 \mathrm{~K}$. This corresponds to the calibration on a sky source which would be used in flight. (In principle one could attempt to omit even this, using measured values of the throughput of the optical chain to convert absorbed power to incident power, for a fully physically based calibration).

The top panel, while showing reasonable agreement, exhibits considerable scatter. The majority of this appears to come from slight differences between the behaviour of the system in the blanked run (PFM5) used to determine $R^{*}$ and $T_{g}$, and the optical run (PFM4). This can be clearly seen as scatter in the bolometer heat-sink temperatures under minimal optical loading. We believe that this is due to small changes in the gain of the readouts (at the level of a few percent), and can compensate for it by taking measurements at the lowest optical loads and adjusting the $T_{g}$ values slightly (up to $0.5 \%$ ) to bring the bolometer temperature into agreement. Ideally this would be carried out under zero optical load, since then the dark pixels will be at the same temperature as the other pixels. However, with the CBB at the lowest temperature, there is some load on the PMW and PLW arrays, and of course in flight there will be loading from the telescope even when viewing blank sky. We can allow for this if the optical power is known. In the case of the $\mathrm{CBB}$, we can calculate the power from its temperature. If such a correction is necessary in flight, we could use the mean of the power measured by the pixels before

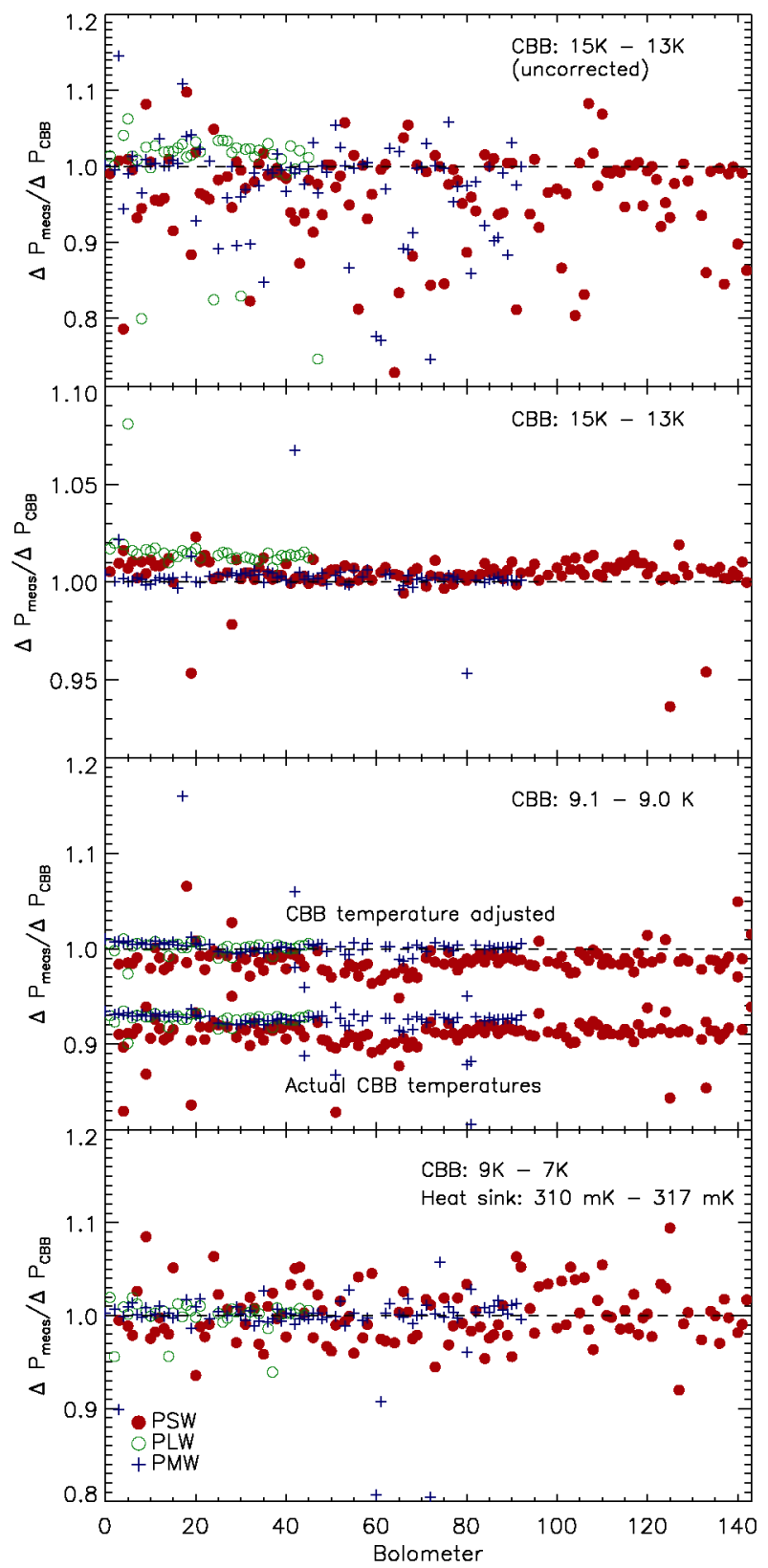

FIGURE 4. Agreement between measured power and power from the $\mathrm{CBB}$, as described in the text, for pairs of load curves at different CBB temperatures as shown in each panel. Results in the lower three panels are corrected as described in the text. Results in the bottom panel were taken at significantly different heat sink temperatures as shown.

correction. This of course makes the assumption that the change in parameters is truly random, as it will remove any change that is common to all the channels.

The corrected results show much better agreement; in the remainder of this paper, we show corrected results. A good test of the model is to compare measurements taken at similar CBB temperatures, since the effect of absolute errors becomes large for small differences in 


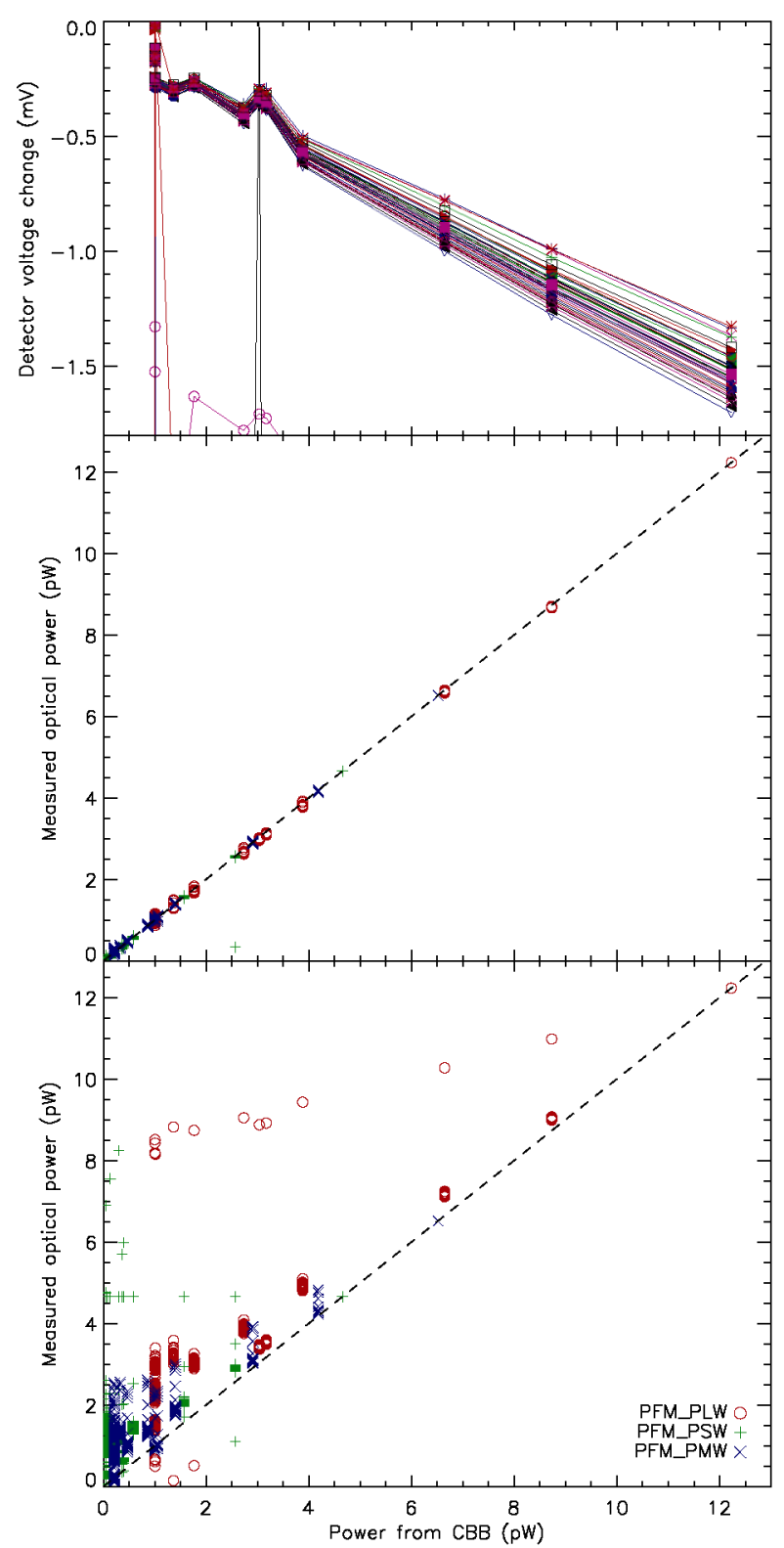

FIGURE 5. Top panel: Change in detector voltage (at operating bias) between different CBB powers and the lowest power, for the PLW array. Lower panels: Measured power as a function of CBB power, showing all pixels apart from outliers as described in the text. The bottom panel shows results without allowing for changes in heat-sink temperature between measurements (obtained by artificially setting the heat-sink temperature to the same value for each load curve, rather than using temperature measured by a dark pixel).

power. This is shown in the third panel in Fig. 4 (CBB temperatures of 9.0 and $9.1 \mathrm{~K}$, points labelled "Actual CBB temperatures"). The systematic error appears to be due to calibration errors in the CBB thermometers; good agreement (points labelled "CBB temperature adjusted") is produced by altering one of the temperatures by $0.1 \%$, which is much smaller than the accuracy of the thermometers.

The bottom panel shows how well changes in heatsink temperature are compensated for; if we didn't allow for this, we would obtain huge errors (values of approximately $0.8,0.4$ and -0.6 for PSW, PMW and PLW respectively when plotted in the same form as Fig. 4). The top panel in Fig. 5 shows the detector voltage change as a function of $\mathrm{CBB}$ power; the deviations from a smooth line are due to variations in heat-sink temperature.

With a model based calibration we are of course not restricted to comparing measurements; we can measure the absolute power for a single measurement. Figure 5 shows the measured power for the range of $\mathrm{CBB}$ powers applied; the agreement can be seen to be excellent. We have removed a few "outlying" bolometers from these results (these make up 13\%,10\% and 9\% of the total number of pixels for PLW, PMW and PSW respectively). We believe that the majority of these outliers are due to errors in automatically deriving the model parameters, and that acceptable parameters could be produced with manual intervention. The bottom panel shows the rather large errors that result if changes in heat-sink temperature are not allowed for.

\section{CONCLUSIONS}

We have shown from measurements taken on SPIRE during ground tests with the flight electronics that a model based calibration produces accurate measurements of the bolometer absorbed power. It remains to be shown that this is also true in flight. However, if this method can be used, it offers several advantages over the traditional empirical calibration. Secondary calibration sources are not required in order to measure non-linearity. The effect of any fluctuations or drift in the heat sink temperature are allowed for automatically, rather than requiring separate empirical corrections. Furthermore, measurements taken at different times can be directly compared even if taken with different heat sink temperatures. Finally, it offers an absolute measurement of flux for a given measurement, rather than just the difference in flux between two observations as has been the case with all sub-mm instruments to date.

\section{REFERENCES}

1. G. L. Pilbratt, Proc. SPIE 7010, 701002 (2008).

2. R. V. Sudiwala, M. J. Griffin, and A. L. Woodcraft, Int. J. Inf. Mill. Waves 23, 545-573 (2002).

3. A. L. Wooderaft, H. Nguyen, M. Griffin, B. Schulz, B. Sibthorpe, and B. Swinyard, Proc. SPIE 7020, 70200F (2008). 